\title{
Home risk factors for childhood pneumonia in Nanjing, China
}

\author{
ZHENG XiaoHong ${ }^{1}$, QIAN Hua ${ }^{1 *}$, ZHAO YiLi ${ }^{1}$, SHEN HongPing ${ }^{1}$, ZHAO ZhuoHui ${ }^{2}$, \\ SUN YueXia ${ }^{3} \&$ SUNDELL Jan ${ }^{1,4}$
}

${ }^{1}$ School of Energy and Environment, Southeast University, Nanjing 210096, China;

${ }^{2}$ School of Public Health, Fudan University, Shanghai 200032, China;

${ }^{3}$ School of Environmental Science and Engineering, Tianjin University, Tianjin 300072, China;

${ }^{4}$ Department of Building Science and Technology, Tsinghua University, Beijing 100084, China

Received October 15, 2012; accepted December 14, 2012; published online April 8, 2013

\begin{abstract}
Pneumonia is a major cause of childhood morbidity in China. Many studies of rural areas have found an association between pneumonia and air pollution from burning of bio-mass. The present study is of children living in urban, modern homes without burning of bio-mass. In order to investigate potential home risk factors associated with children's pneumonia, China Child Home Health $(\mathrm{CCHH})$ questionnaires were randomly distributed to parents of 6461 children in 23 kindergartens in all 11 districts of Nanjing, covering urban, suburban, and industrial areas. The 4014 valid questionnaires (response rate $65.7 \%$ ) were returned and have been analyzed. The lifetime incidence of pneumonia in Nanjing children 1-8 years old is $26.7 \%$. The incidence of croup, asthma and frequent common colds was strongly associated with pneumonia, as was a history of allergies in the family, and persons other than parents (for example grandparents or nanny) taking care of the child. Several home environmental factors, namely, dampness, lack of ventilation, using coal or natural gas (electricity as reference) for cooking, new furniture, and "modern" floor and wall covering materials were also significantly associated with pneumonia. The indoor environment typical of modern apartments in China was a risk factor for pneumonia among children.
\end{abstract}

ventilation, dampness, life style, pneumonia

Citation: Zheng X H, Qian H, Zhao Y L, et al. Home risk factors for childhood pneumonia in Nanjing, China. Chin Sci Bull, 2013, 58: 4230-4236, doi: 10.1007/ s11434-013-5686-5

Childhood pneumonia is the world's leading causes of death among children [1-3], with about 800000 deaths annually of children $<5$ years of age [4,5]. Pneumonia is also the leading cause of death in children $<5$ years old in China [6] and one of the leading causes of death in adults in China [7]. The Chinese Ministry of Health reported that pneumonia mortality of infants was $21.86 / 100 \mathrm{k}$ for children $<1$ year of age and 2.38/100 k for children 1-5 years old in 2009 [6]. Zhang [8] reviewed the literature on childhood pneumonia in China and found that the incidence of pneumonia for children $<5$ years old was $26.5 \%$ (95\% CI: $26.3 \%-26.7 \%$ ) south of the Yangtze River, and 8.4\% (95\% CI: 8.2\%-8.7\%) north of Yangtze River. Zhang [8] and Guan et al. [9] found

*Corresponding author (email: Keenwa@gmail.com) no significant differences in childhood pneumonia incidence between the West and East of China. By comparison, the incidence of pneumonia and acute respiratory infections is quite low in developed countries [10]. It is reported that the incidence of childhood pneumonia is only $0.02 \%$ to $0.38 \%$ episodes per child-year among children $<5$ years old in Norway, USA, Finland, Scotland and UK [11-14], while it was reported that to be $6 \%-27 \%$ episodes per childe-year among children $<5$ years in China [9]. The same conclusions were found by Rudan et al. [3] that the incidence estimated to be 0.29 episodes per child-year in developing and 0.05 episodes per child-year in developed countries among children $<5$ years.

Indoor air pollution from burning unprocessed biomass and coal for cooking has been considered as a major risk 
factor for pneumonia in developing countries [15-17]. Children exposed to smoke from household biomass fuel have been shown to have a rate of acute lower respiratory infection (ALRI) twice that of unexposed children [16]. Zhou [18] compared pneumonia rates in rural and urban settings in Guangzhou and found that pneumonia rates in rural areas were more than four times higher than those in urban areas for children $<1$ year of age, and more than six times higher for children $<5$ years of age. Thus, heavily polluted indoor air is a main cause of acute increased morbidity and mortality consequent to respiratory infections (ARI) [2,19]. Besides of air pollution, Rudan et al. [3] also found that malnutrition, low birth weight, non-exclusive breastfeeding, lack of measles immunization and crowding were risk factors for pneumonia.

China has developed rapidly in the last three decades. In Nanjing, people mainly live in apartments without burning bio-mass or coal. However, the existing literature indicates that the incidence of childhood pneumonia is much higher in cities in China than in developed countries $[9,15]$. The aim of this study was to characterize those indoor aspects of modern Chinese homes that are associated with childhood pneumonia especially to investigate the effect of pollutants generated by modern Chinese homes to childhood pneumonia.

\section{Methods}

\subsection{Questionnaire}

Our study is part of the China, Children, Homes, and Health $(\mathrm{CCHH})$ project, which is investigating associations between home indoor environmental factors and children's health. This is a population-based cross-sectional study. A questionnaire survey was used to investigate health outcomes and home environmental exposure of children less than 8 years old in Nanjing. The questionnaire was consistent with ISAAC (International Study of Asthma and Allergies in Childhood) especially for the questions with children's health. Except for questions that were modified to properly characterize Chinese building characteristics [20], questions are the same as used in Sweden [21], Bulgaria [22] and USA [23] studies. The related questions can be found in Supporting Information.

\subsection{Study site}

Nanjing, the capital of Jiangsu, is located in the Yangtze River Delta economic zone, on the Yangtze River. It has long been one of the most important and developed cities in China. The city has 8 urban districts, 2 suburban districts, 1 industrial district and 2 counties; see Figure 1. All urban districts and 1 suburban district are located south of the Yangtze River, while 1 suburban district and 1 industrial district are located north of the Yangtze River. The climate

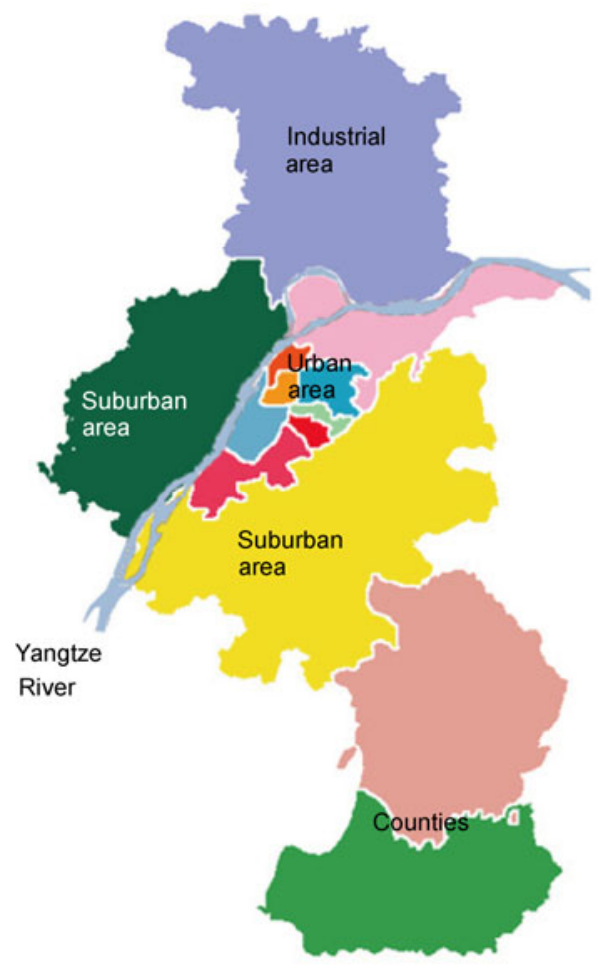

Figure 1 Sketch map of Nanjing showing the urban, suburban, and industrial areas and counties.

is typically hot, humid in summer and cold, humid in winter. Split air conditioners are widely used in Nanjing, both for summer cooling and winter heating.

\subsection{Study populations}

Twenty-three kindergartens were randomly selected in the 11 districts. No kindergartens were selected in the 2 counties. Questionnaires were distributed by kindergarten teachers to children less than 8 years old as homework for their parents. The children submitted the completed questionnaires to teachers. The survey was performed and completed from December 2010 to March 2011 in Nanjing.

\subsection{Data analysis}

Statistical analysis was conducted using SPSS for Windows (SPSS Incorporated, SPSS Release 17.0). Binary logistic models were used to test associations between home environmental exposure, building characteristics, life style and the risk of pneumonia infections. Odds ratios were calculated with $95 \%$ confidence intervals $(\mathrm{CI}) . P$-value less than 0.05 indicated a statistically significant level. For those factors that reached significance in binary analysis, a multivariate logistic model was applied to calculate adjusted odds ratios for pneumonia infections. Step forward elimination techniques were used in the multivariate logistic regression model. 


\section{Results}

Total 6461 questionnaires were distributed and 4014 properly filled-out questionnaires were returned, giving a response rate of $65.7 \%$. The response rate was $61.8 \%$, $68.4 \%$ and $82.0 \%$ for kindergartens in urban, suburban, and industrial areas respectively. We used the kindergarten location as an indication of the location of children's home. Children's homes are usually within walking distance to the kindergarten. Table 1 shows demographic information for investigated children.

The lifetime incidence (at least one incidence) of children's pneumonia in Nanjing is $26.7 \%$ (95\% CI: $25.3 \%-$ $28 \%$ ), $27.2 \%$ (95\% CI: $25.3 \%-29.1 \%$ ), $25.8 \%$ (95\% CI: $23.7 \%-28 \%$ ) and $27.2 \%$ (95\% CI: $22.4 \%-32 \%$ ) for the whole city, urban area, suburban and industrial area respectively. There was no significant difference between locations $(P=0.642)$.

Figure 2 shows crude OR for pneumonia by gender, age and family history of allergy. A family history of allergy is strongly and positively associated with doctor diagnosed pneumonia. Boys have had significantly more pneumonia than girls. Lifetime incidence of pneumonia was not significantly different between children younger than 5 years and older than 5 years old. Thus, young children have a greater incidence for pneumonia.

Table 1 Demographic information of investigated children $(n=4014)$

\begin{tabular}{lccccc}
\hline & & $\begin{array}{c}\text { Total } \\
(\%)\end{array}$ & $\begin{array}{c}\text { Urban } \\
(\%)\end{array}$ & $\begin{array}{c}\text { Suburban } \\
(\%)\end{array}$ & $\begin{array}{c}\text { Industrial } \\
(\%)\end{array}$ \\
\hline \multirow{2}{*}{ Gender } & Male & 51.2 & 27.3 & 19.4 & 4.50 \\
& Female & 48.8 & 25.4 & 19.5 & 3.90 \\
\multirow{3}{*}{ Age } & $<3$ & 1.1 & 1.0 & 0.0 & 0.07 \\
& $3-4$ & 35.1 & 22.1 & 10.5 & 2.40 \\
& $5-6$ & 50.1 & 24.9 & 21.3 & 4.00 \\
& $7-8$ & 13.8 & 4.9 & 7.1 & 1.80 \\
\hline
\end{tabular}

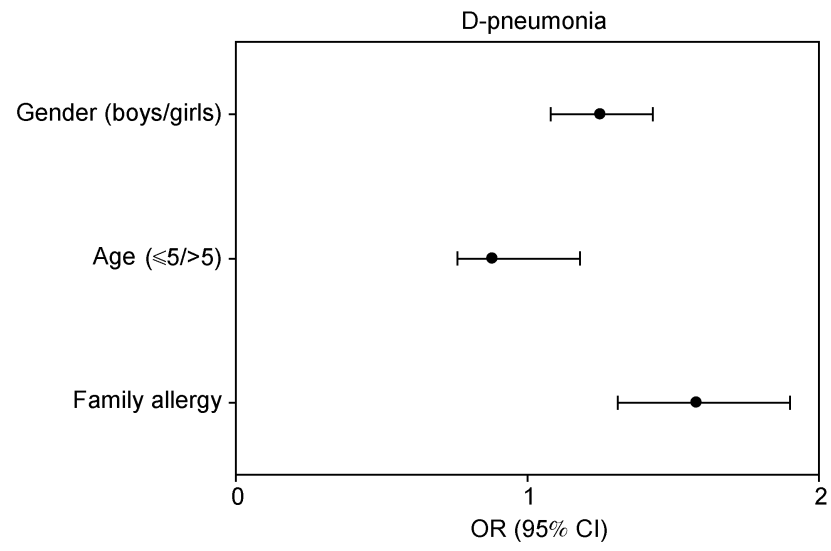

Figure 2 Association between gender, age and family member allergy to diagnosed pneumonia. Variables after slash are used as references in the $Y$-axis.
For lifestyle factors (Figure 3(a)), children with long period of breast feeding ( $>6$ months) reported less pneumonia (OR: 0.81, 95\% CI: 0.70-0.93). Daytime child care before the child's kindergarten life by someone other than parents was a risk factor 1.26 (95\% CI: 1.10-1.46). Compared to using electricity as fuel for cooking, using coal or natural gas was positively associated with pneumonia, for which OR's are 1.75 (95\% CI: 1.16-2.64) and 1.26 (95\% CI: 1.03-1.54) respectively. Figure 3(b) shows odds ratios for associations between building characteristic factors and pneumonia. Home ownership, larger living space $\left(>75 \mathrm{~m}^{2}\right)$ and new furniture were significantly associated with pneumonia $(P<0.05)$. Floors covered by real or laminated wood, and walls covered with paper or emulsion paint were significant risk factors compared to cement $(P<0.05)$. Figure 3(c) shows odds ratios for associations between home ventilation characteristics/odors and pneumonia. Children in homes with smoke removal devices including kitchen fans and hoods, bathroom ventilators, and air conditioner had an increased risk of pneumonia. The perception of pungent odors, dry air, wet air and smells of tobacco smoke in children's room in the previous 3 months also had a significant, positive association with pneumonia. Similar findings were obtained for perception of odors in children's rooms when children were born (data not shown). Figure 3(d) shows odds ratios for associations between indicators of home dampness and pneumonia. Water condensation on windowpanes (both $<5 \mathrm{~cm}$ of height and $>5 \mathrm{~cm}$ of height) at the bottom of windows during wintertime, visible mould, and visible damp stains were significantly and positively associated with children's pneumonia.

Figure 4 shows odds ratios for associations between both other diseases and antibiotic use and diagnosed pneumonia. All diseases except otitis are significantly and positively associated with diagnosed pneumonia; this is especially true for respiratory diseases. Using antibiotics during children aged 0-12 months is also strongly associated with diagnosed pneumonia (OR: 2.70, 95\% CI: 2.19-3.32). Table 2 shows the association between diagnosed pneumonia and the number of times using antibiotics by children aged 0-12 months. Risk increased with more antibiotic was used.

Factors which reached significance level in bivariate analyses were used in the multivariate logistic regression model. Results are shown in Figure 5. Frequent tobacco smell, history of family allergy, child care by grandparents or nanny, smoke removal in kitchen and visible damp stains were found to be most significantly associated with pneumonia.

The crude OR's for these variables were adjusted for gender, age distribution, breast feeding, who was responsible for child care children before kindergarten, coal and gas cooking fuel, ownership of the living space, living space area, new furniture, floor covering materials, wall covering materials, ventilator in bathroom, air conditioner, pungent smell, dry air perception, tobacco smell frequently, conden- 

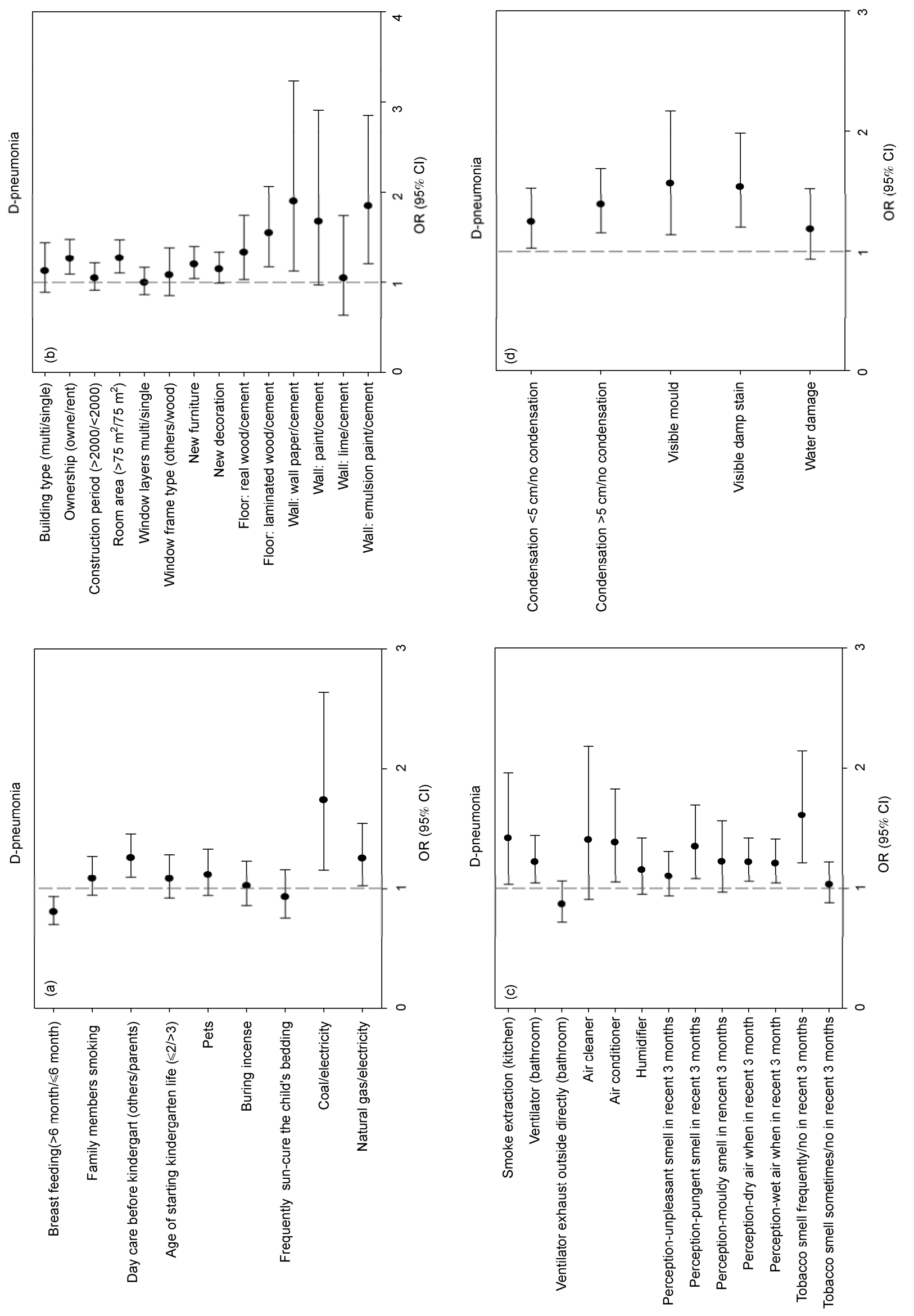


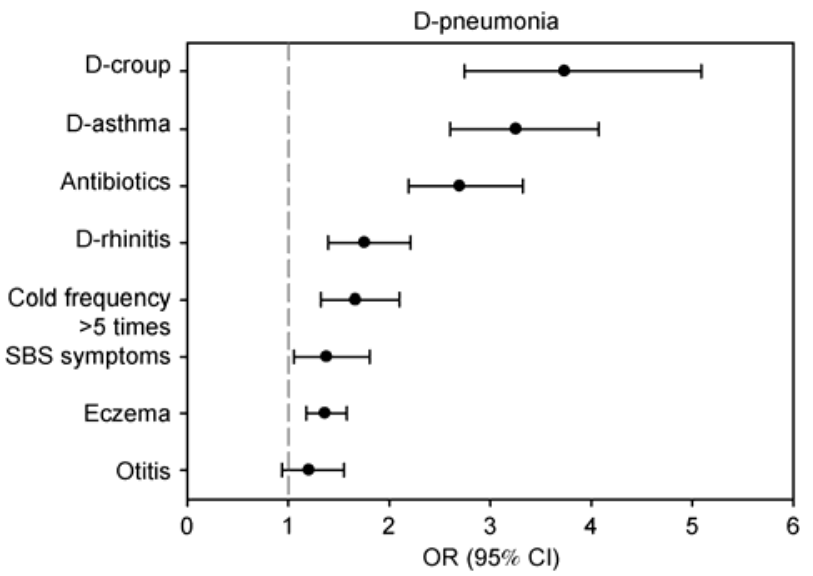

Figure 4 Other diseases associated with diagnosed pneumonia in children in Nanjing.

Table 2 Association between diagnosed pneumonia and times of using antibiotic during children aged $0-12$ months

\begin{tabular}{cccc}
\hline Times of using antibiotic & Crude OR & $95 \% \mathrm{CI}$ & $P$ \\
\hline Never & 1 & 1 & - \\
1 & 1.41 & $1.15-1.72$ & 0.001 \\
2 & 2.02 & $1.61-2.58$ & $<0.01$ \\
3 or more & 2.55 & $2.08-3.14$ & $<0.01$ \\
\hline
\end{tabular}

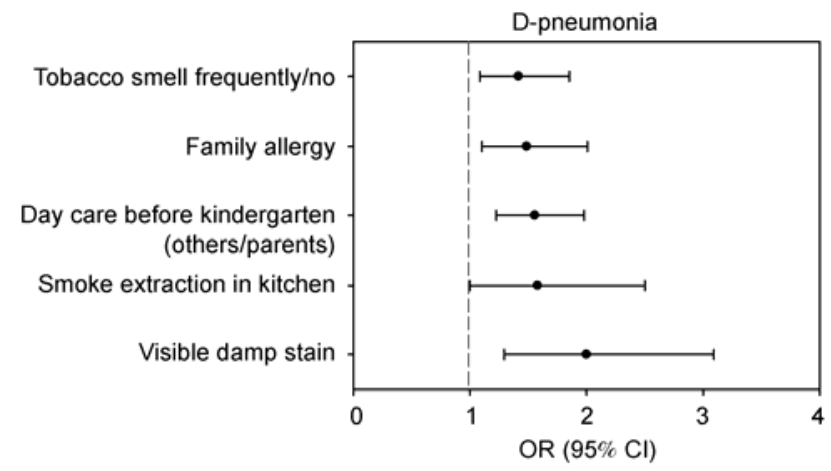

Figure 5 aOR for different factors with pneumonia. Variables after slash were used as references in $Y$-axis.

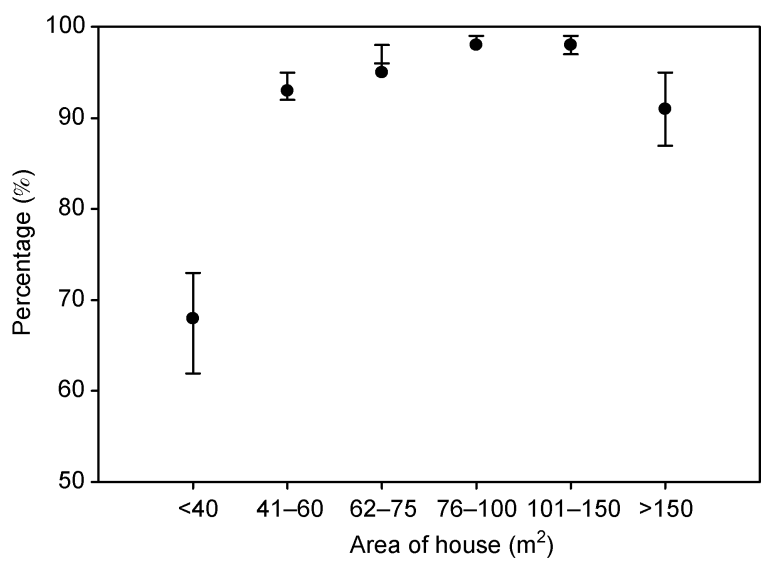

Figure 6 The percentage and 95\% CI of family with smoke removal in kitchen. sation at the bottom of windows, visible mould and visible damp stain.

\section{Discussion}

The sample size in this study was relatively large with a reasonable good response rate $(65.7 \%)$. Except for two outlying counties, all districts were included. This study shows that the lifetime incidence of childhood pneumonia was high among children living in modern apartments, without burning of biomass. The lifetime incidence of diagnosed pneumonia in our survey, $26.7 \%$, is close to the $24.8 \%$ reported for Liyang, a county near to Nanjing in 1994 [24].

We found that most Nanjing families used natural gas (74.5\%) and electricity (33.4\%) for cooking. Few families used coal $(3.1 \%)$ and very few families used biomass $(0.4 \%)$. Nonetheless, we still found coal to be a significant risk factor for pneumonia, consistent with previous studies [3,15-17]. However, natural gas was also a risk for pneumonia. Thus, using electricity for cooking could significantly reduce the risk of pneumonia.

Child care by parents before kindergarten was associated with less reported pneumonia compared to child care by grandparents or nanny. Table 3 suggests that the association may be biased by socio-economic factors. The percentage for children taken by grandparents or nanny lived in larger, newer, new decorated house with new furniture is much higher than children taken by parents. Furthermore, most children taken by parents have breast feeding period $>6$ months, while, most of children taken by grandparents or nanny have breast feeding period $<6$ months.

Modern apartments tend to have construction and decorative materials that we found to be significantly and positively associated with pneumonia. These materials include new furniture, floors covered by wood or laminated wood, walls covered by paper or emulsion paint. Whether and

Table 3 Percentage of children cared for by parents or grandparents/ nanny compared with factors related to socio-economic status

\begin{tabular}{lcccc}
\hline & & \multicolumn{2}{c}{ Take care by } & \multirow{2}{*}{$P$} \\
\cline { 3 - 4 } & & $\begin{array}{c}\text { Parents } \\
(\%)\end{array}$ & $\begin{array}{c}\text { Grandparents } \\
\text { or nanny }(\%)\end{array}$ & \\
\hline Room size & $<75 \mathrm{~m}^{2}$ & 59.5 & 40.5 & $<0.001$ \\
& $>75 \mathrm{~m}^{2}$ & 51.5 & 48.5 & \\
Construction & Before 2000 & 56.8 & 43.2 & \multirow{2}{*}{0.016} \\
period & After 2000 & 52.9 & 47.1 & \\
New furniture & No & 58.7 & 41.3 & $<0.001$ \\
\multirow{2}{*}{ New decoration } & Yes & 52.8 & 47.2 & $<0.01$ \\
\multirow{2}{*}{ Breast feeding } & No & 58.0 & 42.0 & \\
period & Yes & 50.3 & 49.7 & $<0.001$ \\
\hline
\end{tabular}


which pollutants emitted from such materials increase the risk of pneumonia will be addressed in Phase 2 of this study.

Our survey of the indoor environment showed that dampness, smell and cooling by air conditioning were significant risk factors for childhood pneumonia. In Nanjing, almost all air conditioners are split air conditioners, which do not deliver fresh air but rather cool, recirculated air. For reasons of energy savings and thermal comfort, people usually close doors and windows tightly when the air conditioners are running. Thus, air conditioner use tends to reduce ventilation. In the winter, outdoor air temperature and absolute humidity is low. If the building is sealed very well, moisture from humans and other activities, such as cooking and bathing, will accumulate and condense on the windows or walls. Lack of ventilation might induce dampness and smell, which are risk factors of pneumonia. Our findings are consistent with those in the literature that higher ventilation rate could decrease the risk of respiratory infectious diseases [25].

Kitchen smoke removal equipment was also found to be strongly and positively associated with pneumonia. However, smoke removal can decrease aerosols and airborne pollutants in the kitchen. Figure 6 shows that a low percentage of small area homes have smoke removal in the kitchen. For such small area homes without smoke extraction, there is seldom cooking. There is about $14.6 \%$ rented house, $7.8 \%$ non decorated house and $10.0 \%$ non new furniture placed house without kitchen removal equipment, while there is only $1.9 \%$ owned house, $2.9 \%$ new decorated house and $3.9 \%$ new furniture placed house without kitchen removal equipment respectively. Cooking removal equipment seems to be associated with socio-economic factors.

Compared to renting, owning a home was positively associated with pneumonia. $46.7 \%$ of families who own their home have new decoration and $68.2 \%$ families have new furniture, while only $31.2 \%$ families who rent their homes have new decoration and $48.7 \%$ have new furniture (both $P<0.001)$. Furthermore, the finding that larger home size is also a more risk for pneumonia may be due to new furniture and decoration. $66.9 \%$ families with home area $>75 \mathrm{~m}^{2}$ have new furniture and $45.6 \%$ have new decoration, while $54.7 \%$ families with home area $<75 \mathrm{~m}^{2}$ have new furniture and $36.1 \%$ have new decoration. This is a further indication that modern housing may have factors which increase the risk of pneumonia.

Although $63.2 \%$ of the families in our survey had a member who smokes, we found no significant association between family members smoking and pneumonia. Tobacco smell, however, showed a significant association with pneumonia. The reason may be that smokers do not smoke indoors especially in a room with a child, which reduces the smoking smell as shown in Table 4. However, it should be noted that the tobacco smell was significantly associated with family member smoking (OR: 10.99, 95\% CI: 8.94
Table 4 Percentage of ETS odor in room with/without family member smoking

\begin{tabular}{cccc}
\hline \multirow{2}{*}{$\begin{array}{c}\text { Family } \\
\text { member smoking }\end{array}$} & \multicolumn{3}{c}{ Tobacco smell (\%) } \\
\cline { 2 - 4 } & Frequently & Sometimes & Never \\
\hline Yes & 9.3 & 41.1 & 49.6 \\
No & 0.7 & 7.7 & 91.5 \\
\hline
\end{tabular}

13.51).

In this study, we found that the use of antibiotics was strongly associated with diagnosed pneumonia (Table 2). Considering the duration of pneumonia, the association between lengths of antibiotic use with pneumonia might indicate that diagnosed pneumonia was treated by antibiotics. Asthma and allergies, croup, rhinitis, SBS symptoms, eczema and cold frequently are strongly associated with pneumonia, as shown in Figure 4.

\section{Conclusions}

This paper investigated the association between childhood pneumonia and indoor environment factors in modern homes. The pneumonia incidence is found to be high in Nanjing. Lack of ventilation, gas as cooking fuel, dampness, new furniture, "modern" floor and wall covering materials show significant associations with the incidence of pneumonia. Other factors such as family allergy, child care by non-parents, other respiratory diseases were also reported to be associated with pneumonia. In summary, modern life style and home environment play an important role in developing pneumonia infections among children in Nanjing.

This work was supported by the National Natural Science Foundation of China (51008063).

1 Bryce J, Boschi-Pinto C, Shibuya K, et al. WHO estimates of the causes of death in children. Lancet, 2005, 365: 1147-1152

2 Harris A M, Sempertegui F, Estrella B, et al. Air pollution and anemia as risk factors for pneumonia in Ecuadorian children: A retrospective cohort analysis. Environ Health-Glob, 2011, 10: 1-8

3 Rudan I, Boschi-Pinto C, Biloglav Z, et al. Epidemiology and etiology of childhood pneumonia. Bull World Health Organ, 2008, 86: 408416

4 World Health Organization, UNICEF. Pneumonia: The forgotten killer of children. Geneva: World Health Organization, 2006

5 World Health Organization. Pneumococcal conjugate vaccine for childhood immunization-WHO position paper. Wkly Epidemiol Rec, 2007, 82: 93-104

62010 China Health Statistical Yearbook. Ministry of Health of the People's Republic of China, 2010

7 He J, Gu D F, Wu X G, et al. Major causes of death among men and women in China. New Engl J Med, 2005, 353: 1124-1134

8 Zhang T. Study on the epidemiology of children pneumonia, evaluation of the etiologic diagnosis method, and exploring multiplex PCR method for determining capsular serotypes of pneumococci (in Chinese). Doctoral Dissertation. Shanghai: Fudan University, 2010

9 Guan X, Silk B J, Li W, et al. Pneumonia incidence and mortality in 
Mainland China: Systematic review of Chinese and English literature, 1985-2008. PLoS One, 2010, 5: e11721

10 Prayle A, Atkinson M, Smyth A. Pneumonia in the developed world. Paediatr Respir Rev, 2011, 12: 60-69

11 Roxburgh C S, Youngson G G, Townend J A, et al. Trends in pneumonia and empyema in Scottish children in the past 25 years. Arch Dis Child, 2008, 93: 316-318

12 Senstad A C, Suren P, Brauteset L, et al. Community-acquired pneumonia (CAP) in children in Oslo, Norway. Acta Paediatr, 2009, 98: $332-336$

13 Myles P R, Hubbard R B, Gibson J E, et al. Pneumonia mortality in a UK general practice population cohort. Eur J Public Health, 2009, 19: 521-526

14 Myles P R, McKeever T M, Pogson Z, et al. The incidence of pneumonia using data from a computerized general practice database. Epidemiol Infect, 2009, 137: 709-716

15 The Lancet. Indoor air pollution and acute respiratory infections in children. Lancet, 1992, 339: 396-398

16 Smith K R, McCracken J P, Weber M W, et al. Effect of reduction in household air pollution on childhood pneumonia in Guatemala (RESPIRE): A randomised controlled trial. Lancet, 2011, 378: 17171726

17 Dherani M, Pope D, Mascarenhas M, et al. Indoor air pollution from unprocessed solid fuel use and pneumonia risk in children aged under five years: A systematic review and meta-analysis. Bull World Health
Organ, 2008, 86: 390-398

18 Zhou Y. Seasonal analysis of the morbidity of pneumonia in children under 5 years of age in Guangzhou (in Chinese). Chin J Health Care, 2000, 8: 35-36

19 Samet J M, Dominici F, Curriero F C, et al. Fine particulate air pollution and mortality in 20 US Cities, 1987-1994. New Engl J Med, 2000, 343: 1742-1749

20 Sun Y, Wang Z, Zhang Y, et al. In China, students in crowded dormitories with a low ventilation rate have more common colds: Evidence for airborne transmission. PLoS One, 2011, 6: e27140

21 Sun Y, Zhang Y, Bao L, et al. Ventilation and dampness in dorms and their associations with allergy among college students in China: A case-control study. Indoor Air, 2011, 21: 277-283

22 Naydenov K, Popov T, Mustakov T, et al. The association of pet keeping at home with symptoms in airways, nose and skin among Bulgarian children. Pediat Allerg Imm-UK, 2008, 19: 702-708

23 Sun Y, Sundell J. Early daycare attendance increase the risk for respiratory infections and asthma of children. J Asthma, 2011, 48: 790796

24 Cheng P. Reducing Mortality rate of childhood pneumonia by ARI management (in Chinese). Jiangsu Journal of Preventive Medicine, 1996, 2: 48-49

25 Li Y, Leung G M, Tang J W, et al. Role of ventilation in airborne transmission of infectious agents in the built environment-A multidisciplinary systematic review. Indoor Air, 2007, 17: 2-18

Open Access This article is distributed under the terms of the Creative Commons Attribution License which permits any use, distribution, and reproduction in any medium, provided the original author(s) and source are credited.

\section{Supporting Information}

\section{Questions in the questionnaries related to this paper}

The supporting information is available online at csb.scichina.com and www.springerlink.com. The supporting materials are published as submitted, without typesetting or editing. The responsibility for scientific accuracy and content remains entirely with the authors. 